\title{
Provably Secure Key-Updating Schemes in Identity-Based Systems
}

\author{
S. Shinozaki $\dagger \quad$ T. Itoh $\ddagger \quad$ A. Fujioka $\dagger \quad$ S. Tsujii $\dagger$ \\ $\dagger$ Department of Electrical and Electronic Engineering \\ Faculty of Engineering \\ Tokyo Institute of Technology \\ O-okayama, Meguro-ku, Tokyo 152, Japan \\ $\ddagger$ Department of Information Processing \\ The Graduate School at Nagatsuta \\ Tokyo Institute of Technology \\ 4259 Nagatsuta, Midori-ku, Yokohama 227, Japan \\ e-mail: titoh@cc.titech.ac.jp
}

\begin{abstract}
:
In this paper, we present Key-Updating Schemes in identity-based (identification or signature) systems, and consider the security of the schemes. We propose two kinds of key-updating schemes, i.e., one is sequential type and the other is parallel type, and show that both schemes are equivalent to each other in a polynomial time sense, i.e., there exists a deterministic polynomial time algorithm that transforms the sequential key-updating scheme to the parallel one, and vice versa. We also show that even if any polynomially many entities conspire to find a secret-key of any other entities, both key-updating schemes are provably secure against polynomially many times key-updating if decrypting RSA is hard.
\end{abstract}




\section{Introduction}

In identity-based systems, each entity $i$ has his(her) own identity number $I D_{i}$, and a trusted center needs to generate a pair of a public information $P$ (known to all entities) and a secret information $S$ (known to only the trusted center), and a pair of public-key $P K_{i}$ and secret-key $S K_{i}$ for entity $i$. Let a probabilistic polynomial time algorithm $C K G$ be a center-key generator that, on input $1^{k}$, outputs a pair of the public information $P\left(|P|=O\left(k^{c}\right)\right.$ for some constant $\left.c>0\right)$ and the secret information $S\left(|S|=O\left(k^{d}\right)\right.$ for some constant $d>0$ ), i.e., $C K G\left(1^{k}\right)=\langle P, S\rangle$, and let a probabilistic polynomial time algorithm $E K G$ be a entity-key generator that, on input $1^{k}, P, S$, and $I D_{i}$, outputs a pair of public-key $P K_{i}$ and secret-key $S K_{\mathrm{i}}$ for entity $i$, i.e., $E K G\left(\left\langle 1^{k}, P, S, I D_{\mathrm{i}}\right\rangle\right)=$ $\left\langle P K_{i}, S K_{i}\right\rangle$. Note that $k$ is the security parameter.

When a foolish entity $j$ carelessly loses his secret-key $S K_{j}$ or reveals it and asks the trusted center again to generate a new pair of public-key $P K_{j}^{\prime}$ and secret-key $S K_{j}^{\prime}$ for him, what should the trusted center do? If the system is provably secure (see, e.g., [FS], [FFS], [GQ], [OO].), i.e., there exist no efficient algorithms for entity $j$ to derive the secret information $S$ from $P, I D_{j}$, and a single pair of $\left\langle P K_{j}, S K_{j}\right\rangle$, then (presumably) the simplest and secure way to update the secret-key $S K_{j}$ to $S K_{j}^{\prime}$ is to make the trusted center run $C K G$ on input $1^{k}$ in order to regenerate a new pair of public information $P^{\prime}$ and secret information $S^{\prime}$ and to make the trusted center regenerate a new pair of publickey $P K_{j}^{\prime}$ and secret-key $S K_{j}^{\prime}$ for the entity $j$ by running $E K G$ on input $1^{k}, P^{\prime}, S^{\prime}$, and $I D_{j}$ (or $S K_{j}$ ). This scheme, however, imposes cumbersome procedures on the trusted center and all entities, because the trusted center must regenerate not only a new pair of public-key $P K_{j}^{\prime}$ and secret-key $S K_{j}^{\prime}$ for the foolish entity $j$ but a new pair of public-key $P K_{i}^{\prime}$ and secret-key $S K_{i}^{\prime}$ for every entity $i(\neq j)$.

Another way to update the secret-key $S K_{j}$ to $S K_{j}^{\prime}$ is to make the trusted center run only $E K G$ on input $1^{k}, P, S$, and $I D_{j}$ (or $S K_{j}$ ) and to regenerate a new pair of public-key $P K_{j}^{\prime}$ and secret-key $S K_{j}^{-\prime}$ only for the foolish entity $j$, while those for the other entities $i(\neq j)$ are unchanged. This scheme is much simpler than before, but unfortunately there might be a possibility that the entity $j$ can derive the secret information $S$ efficiently from $P, I D_{j}, P K_{j}, S K_{j}, P K_{j}^{\prime}$, and $S K_{j}^{\prime}$.

Thus this provokes us to construct efficient and provably secure key-updating schemes in identity-based systems in the above sense. To do this, we take the extended FiatShamir scheme [GQ], [OO] as an identity-based system, and apply two kinds of keyupdating schemes, one is sequential and the other is parallel, to the extended Fiat-Shamir scheme. (The details will be discussed in Section 2.) We also show that our key-updating 
schemes are provably secure against polynomially many times key-updating even if any polynomially many entities conspire to find a secret-key of any other entities.

The organization of this paper is as follows: Section 2 presents a brief description of key-generation and key-distribution in the extended Fiat-Shamir scheme [GQ], [OO], and proposes two kinds of key-updating schemes, sequential one and parallel one; Section 3 shows that both schemes are equivalent to each other in a polynomial time sense, i.e., there exists a polynomial time algorithm that transforms the sequential key-updating scheme to the parallel one, and vise versa; Section 4 gives a main result that both keyupdating schemes are provably secure against polynomially many times key-updating, i.e., any polynomially many conspiring entities can not find a secret-key of any other entities under the assumption that decrypting RSA is hard; and Section 5 finally gives conclusion and remarks, and refers to extensions of our results to more general settings and the security of the schemes against conspiracy of entities.

\section{Key-Updating Schemes}

\subsection{Extended Fiat-Shamir Scheme}

This subsection presents a brief description of key-generation and key-distribution in the extended Fiat-Shamir scheme [GQ], [OO]. The extended Fiat-Shamir scheme is an extension of the Fiat-Shamir scheme [FS], [FFS], and is shown, under the assumption that factoring is hard, to be zero-knowledge in the sequential execution (of the protocol) and to be non-transferable in the parallel execution (of the protocol). This scheme is an identity-based system, and thus the trusted center needs to generate a pair of public information $P$ (known to all entities) and secret information $S$ (known to only trusted center) and to distribute a pair of public-key $P K_{i}$ and secret-key $S K_{i}$ for each entity $i$ with his identity number $I D_{i}$, in the following way:

The trusted center has two probabilistic polynomial time algorithms, i.e., center-key generator $C K G$ and entity-key generator $E K G$; On input $1^{k}$, the center-key generator $C K G$ outputs a pair of public information $n(=p \cdot q)$ and secret information $\langle p, q\rangle$, where $p, q \in \mathcal{O P}$ and $|p|=|q|=k$, and on input $1^{k}, n,\langle p, q\rangle$, and $I D_{i}$, the entity-key generator $E K G$ outputs a pair of public-key $e_{i}$ and secret-key $S_{i}$ for entity $i$ such that $S_{i}^{e^{i}} \equiv I D_{i}$ $(\bmod n)$. Note that $\mathcal{O P}$ denotes a set of odd primes and $|a|$ denotes the length of binary encoding of $a$. For details of identification and signature protocols in the extended FiatShamir scheme, see [GQ], [OO]. 


\subsection{Key-Updating Schemes}

In this subsection, we propose two kinds of key-updating schemes, sequential one [FT] and parallel one, in the extended Fiat-Shamir scheme. Consider the case where some entity $i$ asks the trusted center to issue a new pair of public-key $e_{i}^{\prime}$ and secret-key $S_{i}^{\prime}$ for the entity $i$ in some reason, e.g., losing or revealing his original secret-key $S_{i}$.

Informally, our key-updating schemes are as follows: (1) Sequential Key-Updating Scheme (SKU) is a key-updating scheme in which the trusted center runs the entity-key generator $E K G$ on input $1^{k}, n,\langle p, q\rangle, e_{i}$, and $S_{i}$ (instead of $\left.I D_{i}\right)$, and generates a new pair of $\left(e_{i}^{\prime}, S_{i}^{\prime}\right)$ such that $S_{i}^{k_{i}^{\prime}} \equiv S_{i}(\bmod n)$ and $e_{i} \neq e_{i}^{\prime}$, and (2) Parallel Key-Updating Scheme (PKU) is a key-updating scheme in which the trusted center runs the entity-key generator $E K G$ on input $1^{k}, n,\langle p, q\rangle, e_{i}$, and $I D_{i}$, and generates a new pair of $\left\langle e_{i}^{\prime}, S_{i}^{\prime}\right\rangle$ such that $S_{i}^{l e_{i}^{\prime}} \equiv I D_{i}(\bmod n)$ and $e_{i} \neq e_{i}^{\prime}$. Note that for entity $i$, a pair of public-key and secret-key will be $\left\langle e_{i} e_{i}^{\prime}, S_{i}^{\prime}\right\rangle$ in SKU, while will be $\left\langle e_{i}^{\prime}, S_{i}^{\prime}\right\rangle$ in PKU.

This formulation, however, does not necessarily match our desire, because a malicious entity $j$ might ask the trusted center to issue new pairs of $\left\langle e_{j}^{\prime}, S_{j}^{\prime}\right\rangle$ many times for compromising the secret information $\langle p, q\rangle$. Then we formally define our key-updating schemes in more general settings.

Let $U(|n|)$ be any fixed polynomial in $|n|$, and let $\mathcal{O P}(\ell)$ denote a set of odd primes less than $\ell$. Here we assume that each entity $i$ is allowed to ask the trusted center to issue new pairs of $\left\langle e_{i}^{\prime}, S_{i}^{\prime}\right\rangle$ at most $U(|n|)$ times.

\section{Sequential Key-Updating Scheme (SKU):}

Initial Key-Setting Stage: For each entity $i$ (with $I D_{i} \in \mathcal{Z}_{n}^{*}$ ), the trusted center distributes a pair of his public-key $e_{i}^{(0)}$ and his secret-key $S_{i}^{(0)}$ such that $I D_{i} \equiv\left\{S_{i}^{(0)}\right\}^{e_{i}^{(0)}}$ $(\bmod n)$, where $e_{i}^{(0)} \in \mathcal{O P}(\lfloor\sqrt{n} / 4\rfloor), S_{i}^{(0)} \not \equiv D_{i}(\bmod n)$, and $I D_{i}^{2} \not \equiv 1(\bmod n)$.

Key-Updating Stage: For entity $i$ in the $r_{i}$-th $\left(1 \leq r_{i} \leq U(|n|)\right)$ key-updating, the trusted center distributes a new pair of $\left\langle e_{i}^{\left(r_{i}\right)}, S_{i}^{\left(r_{i}\right)}\right\rangle$ such that $S_{i}^{\left(r_{i}-1\right)} \equiv\left\{S_{i}^{\left(r_{i}\right)}\right\}^{e_{i}^{\left(r_{i}\right)}}$ $(\bmod n)$, where $e_{i}^{\left(r_{i}\right)} \in \mathcal{O P}(\lfloor\sqrt{n} / 4\rfloor), e_{i}^{(j)} \neq e_{i}^{\left(r_{i}\right)}\left(0 \leq j<r_{i}\right), S_{i}^{\left(r_{i}\right)} \not \equiv D_{i}(\bmod n)$, and $S_{i}^{(j)} \not \equiv S_{i}^{\left(r_{i}\right)}(\bmod n)\left(0 \leq j<r_{i}\right)$.

Remark 2.1: In the $r_{i}$-th key-updating of SKU, a pair of the public-key and the secret-key will be $\left\langle e_{i}^{(0)} e_{i}^{(1)} \cdots e_{i}^{\left(r_{i}\right)}, S_{i}^{\left(r_{i}\right)}\right\rangle$. The condition $S_{i}^{(j)} \not I D_{i}(\bmod n)\left(0 \leq j \leq r_{i}\right)$ shows that the trusted center avoids distributing trivial secret-key $S_{i}^{(j)}$, and the condition $S_{i}^{(j)} \not S_{i}^{\left(r_{i}\right)}(\bmod n)\left(0 \leq j<r_{i}\right)$ implies that the trusted center does not distribute the same secret-key $S_{i}^{\left(r_{i}\right)}$ again, because old secret-keys might be known to someone else. The trusted center does not care about collisions of secret-keys among entities. 


\section{Parallel Key-Updating Scheme (PKU):}

Initial Key-Setting Stage: For each entity $i$ (with $I D_{i} \in \mathcal{Z}_{n}^{*}$ ), the trusted center distributes a pair of his public-key $f_{i}^{(0)}$ and his secret-key $T_{i}^{(0)}$ such that $I D_{i} \equiv\left\{T_{i}^{(0)}\right\}^{f_{i}^{(0)}}$ $(\bmod n)$, where $f_{i}^{(0)} \in \mathcal{O P}(\lfloor\sqrt{n} / 4\rfloor), T_{i}^{(0)} \not \equiv I D_{i}(\bmod n)$, and $I D_{i}^{2} \not \equiv 1(\bmod n)$.

Key-Updating Stage: For entity $i$ in the $r_{i}$-th $\left(1 \leq r_{i} \leq U(|n|)\right)$ key-updating, the trusted center distributes a new pair of $\left\langle f_{i}^{\left(r_{i}\right)}, T_{i}^{\left(r_{i}\right)}\right\rangle$ such that $I D_{i} \equiv\left\{T_{i}^{\left(r_{i}\right)}\right\}^{f_{i}^{\left(r_{i}\right)}}(\bmod n)$, where $f_{i}^{\left(r_{i}\right)} \in \mathcal{O P}(\lfloor\sqrt{n} / 4\rfloor), f_{i}^{(j)} \neq f_{i}^{\left(r_{i}\right)}\left(0 \leq j<r_{i}\right), T_{i}^{\left(r_{i}\right)} \not I D_{i}(\bmod n), T_{i}^{(j)} \not \equiv T_{i}^{\left(r_{i}\right)}$ $(\bmod n)\left(0 \leq j<r_{i}\right)$, and $I D_{i}^{f_{i}^{(j)} \cdots f_{i}^{\left(r_{i}\right)}} \neq I D_{i}(\bmod n)\left(0 \leq j<r_{i}\right)$.

Remark 2.2: In the $r_{i}$-th key-updating of PKU, a pair of the public-key and the secret-key will be $\left\langle f_{i}^{\left(r_{i}\right)}, T_{i}^{\left(r_{i}\right)}\right\rangle$. The meaning of conditions $T_{i}^{(j)} \not \equiv I D_{i}(\bmod n)\left(0 \leq j \leq r_{i}\right)$ and $T_{i}^{(j)} \not T_{i}^{\left(r_{i}\right)}(\bmod n)\left(0 \leq j<r_{i}\right)$ is similar to the one in the Remark 2.1. The trusted center does not care about collisions of secret-keys among entities.

\section{Transforms Between SKU and PKU}

This section shows that key-updating schemes SKU and PKU are equivalent to each other in a polynomial time sense, i.e., there exists a deterministic polynomial time algorithm that transforms SKU to PKU, and vice versa.

Let $\mathcal{S C}_{k}$ denote a set of strong composites with the security parameter $k$, i.e.,

$$
\begin{aligned}
\mathcal{S C}_{k}=\{n \mid n=p \cdot q, & \neq \neq,|p|=|q|=k, \\
& \left.p=2 p^{\prime}+1, q=2 q^{\prime}+1, p, q, p^{\prime}, q^{\prime} \in \mathcal{O} \mathcal{P}\right\} .
\end{aligned}
$$

To prove that for $n \in \mathcal{S C}_{k}$, key-updating schemes SKU and PKU are deterministic polynomial time transformable to each other, we need to show the following lemmas:

Lemma 3.1: Let $n \in S \mathcal{C}_{k}$. Then for any odd $e$ less than $\lfloor\sqrt{n} / 4\rfloor, e<\min \left\{p^{\prime}, q^{\prime}\right\}$ and $\operatorname{gcd}(e, \lambda(n))=1$, where $\lambda(n)$ is the Carmichael function $[\mathrm{Kr}]$ of $n$.

Proof: From the definition of $\mathcal{S C}_{k}$, it follows that

$$
\lambda(n)=\operatorname{lcm}(p-1, q-1)=\operatorname{lcm}\left(2 p^{\prime}, 2 q^{\prime}\right)=2 p^{\prime} q^{\prime} .
$$

Note that $n \in S \mathcal{C}_{k}$, i.e., $|p|=|q|=k$, then $2 \cdot \min \{p, q\}>\max \{p, q\}$. Hence,

$$
\begin{aligned}
\lfloor\sqrt{n} / 4\rfloor & \leq\lfloor\max \{p, q\} / 4\rfloor \\
& \leq\lfloor\min \{p, q\} / 2\rfloor \\
& =\left\lfloor\left(2 \cdot \min \left\{p^{\prime}, q^{\prime}\right\}+1\right) / 2\right\rfloor \\
& =\left\lfloor\min \left\{p^{\prime}, q^{\prime}\right\}+1 / 2\right\rfloor=\min \left\{p^{\prime}, q^{\prime}\right\},
\end{aligned}
$$


and thus $e<\min \left\{p^{\prime}, q^{\prime}\right\}$. It immediately follows, from the fact that $\lambda(n)=2 p^{\prime} q^{\prime}$, that $\operatorname{gcd}(e, \lambda(n))=\operatorname{gcd}\left(e, 2 p^{\prime} q^{\prime}\right)=1$, because $e<\min \left\{p^{\prime}, q^{\prime}\right\}, e$ is odd, and $p^{\prime}, q^{\prime} \in \mathcal{O P}$.

Lemma 3.2: Let $n \in \mathcal{S C}_{k}$, and $x \in Z_{n}^{*}$ such that $x^{2} \not \equiv 1(\bmod n)$. For any distinct odd numbers $a_{1}$ and $a_{2}, x^{a_{2}-a_{1}} \not \equiv 1(\bmod n)$, where $a_{1}, a_{2}<\lfloor\sqrt{n} / 4\rfloor$.

Proof: By Contradiction. Without loss of generality, we assume that $a_{1}<a_{2}$. Assume that $x^{a_{2}-a_{1}} \equiv 1(\bmod n)$. This implies that the order of $x$ modulo $n$ divides both $\lambda(n)$ and $a_{2}-a_{1}$. Since $n \in \mathcal{S} \mathcal{C}_{k}, \lambda(n)=2 p^{\prime} q^{\prime}$ and $0<a_{2}-a_{1}<\min \left\{p^{\prime}, q^{\prime}\right\}$ (see Lemma 3.1.), and thus the order of $x$ modulo $n$ is equal to either 1 or 2 . This, however, contradicts the assumption that $x^{2} \not \equiv 1(\bmod n)$. Hence $x^{a_{2}-a_{1}} \not \equiv 1(\bmod n)$.

Lemma 3.3: Let $r$ be any positive integer and let $n \in S \mathcal{C}_{k}$. Let $I D \in Z_{n}^{*}, S^{(i)} \in Z_{n}^{*}$ $(0 \leq i \leq r)$, and $e^{(i)} \in \mathcal{O P}(\lfloor\sqrt{n} / 4\rfloor)(0 \leq i \leq r)$ satisfy the relation that $I D \equiv\left\{S^{(0)}\right\}^{e^{(0)}}$ $(\bmod n), S^{(i-1)} \equiv\left\{S^{(i)}\right\}^{e^{(i)}}(\bmod n)(1 \leq i \leq r)$, where $I D^{2} \neq 1(\bmod n)$ and $e^{(i)} \neq e^{(j)}$ $(0 \leq i<j \leq r)$. Then $I D \not \equiv S^{(i)}(\bmod n)(0 \leq i \leq r)$ and $S^{(i)} \not S^{(j)}(\bmod n)(0 \leq i<$ $j \leq r)$ iff $I D^{e^{(i)} \ldots e^{(j)}} \not \equiv I D(\bmod n)(0 \leq i<j \leq r)$.

Proof: Since $n \in S \mathcal{C}_{k}$ and $I D\left(\in Z_{n}^{*}\right)$ satisfies $I D^{2} \not \equiv 1(\bmod n)$, it follows, from Lemma 3.2 , that for $e^{(i)} \in \mathcal{O P}(\lfloor\sqrt{n} / 4\rfloor)(0 \leq i \leq r), I D^{e^{(i)}-1} \not \equiv 1(\bmod n)(0 \leq i \leq r)$, and hence $I D^{e^{(i)}} \not \equiv I D(\bmod n)(0 \leq i \leq r)$. Note that $x^{e} \equiv \dot{y^{e}}(\bmod n)$ iff $x \equiv y(\bmod n)$ for $e$ such that $\operatorname{gcd}(e, \lambda(n))=1$. (see Lemma 3.1.) Then for all $i(0 \leq i \leq r)$,

$$
\begin{aligned}
I D \equiv S^{(i)}(\bmod n) & \Longleftrightarrow I D^{(0) \ldots e^{(i)}} \equiv\left\{S^{(i)}\right\}^{e^{(0) \ldots e^{(i)}}}(\bmod n) \\
& \Longleftrightarrow I D^{e^{(0) \ldots e^{(i)}} \equiv I D(\bmod n) .}
\end{aligned}
$$

On the other hand, for any $i, j(0 \leq i<j \leq r)$, we have

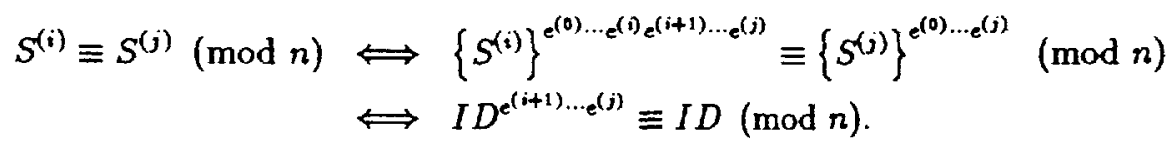

Hence $I D \not \equiv S^{(i)}(\bmod n)(0 \leq i \leq r)$ and $S^{(i)} \not S^{(j)}(0 \leq i<j \leq r)$ iff $I D^{e^{(j) \ldots e^{(j)}} \not I D}$ $(\bmod n)(0 \leq i<j \leq r)$.

Lemma 3.4: Let $r$ be any positive integer and let $n \in S \mathcal{C}_{k}$. Let $I D \in Z_{n}^{*}, T^{(i)} \in Z_{n}^{*}$ $(0 \leq i \leq r)$, and $f^{(i)} \in \mathcal{O P}(\lfloor\sqrt{n} / 4\rfloor)(0 \leq i \leq r)$ satisfy the relation that $I D \equiv\left\{T^{(i)}\right\}^{\mathcal{( n )}^{(i)}}$ $(\bmod n)(0 \leq i \leq r)$, where $I D^{2} \neq 1(\bmod n)$, and $f^{(i)} \neq f^{(j)}(0 \leq i<j \leq r)$. Then $I D \not \equiv T^{(i)}(\bmod n)(0 \leq i \leq r)$ and $T^{(i)} \not T^{(j)}(\bmod n)(0 \leq i<j \leq r)$. 
Proof: Since $n \in S \mathcal{C}_{k}$ and $I D\left(\in Z_{n}^{*}\right)$ satisfies $I D^{2} \not \equiv 1(\bmod n)$, it follows, from Lemma

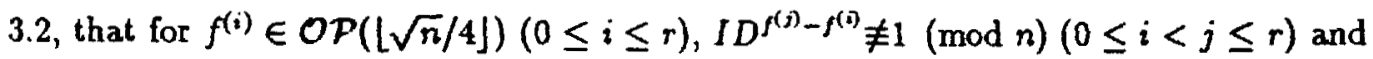
$I D^{f^{(i)}-1} \not \equiv 1(\bmod n)(0 \leq i \leq r)$. Then for all $i(0 \leq i \leq r)$, we have

$$
\begin{aligned}
I D \equiv T^{(i)}(\bmod n) & \Longleftrightarrow I D^{f^{(i)}} \equiv\left\{T^{(i)}\right\}^{f^{(i)}}(\bmod n) \\
& \Longleftrightarrow I D^{f^{(i)}} \equiv I D(\bmod n) \\
& \Longleftrightarrow I D^{f^{(i)}-1} \equiv 1(\bmod n) .
\end{aligned}
$$

On the other hand, for any $i, j(0 \leq i<j \leq r)$, we also have

$$
\begin{aligned}
& T^{(i)} \equiv T^{(j)}(\bmod n) \Longleftrightarrow\left\{\left\{T^{(i)}\right\}^{f^{(i)}}\right\}^{f^{(j)}} \equiv\left\{\left\{T^{(j)}\right\}^{f^{(j)}}\right\}^{f^{(i)}}(\bmod n) \\
& \Longleftrightarrow I D^{f^{(j)}} \equiv I D^{f^{(i)}}(\bmod n) \\
& \Longleftrightarrow I D^{f^{(i)}-f^{(i)}} \equiv 1(\bmod n)
\end{aligned}
$$

hence $I D \not \equiv T^{(i)}(\bmod n)(0 \leq i \leq r)$ and $T^{(i)} \not T^{(j)}(\bmod n)(0 \leq i<j \leq r)$.

Let $U(|n|)$ be any fixed polynomial in $|n|$ and let $r$ be any positive integer not greater than $U(|n|)$. Here we define $\mathcal{C}_{\text {SKU }}$ to be a set of tuples $\left\langle n, I D, S^{(r)}, e^{(r)}\right\rangle$ that satisfy

$$
\begin{aligned}
I D & \equiv\left\{S^{(0)}\right\}^{e^{(0)}}(\bmod n) \\
S^{(i-1)} & \equiv\left\{S^{(i)}\right\}^{e^{(i)}}(\bmod n)(1 \leq i \leq r) \\
I D^{2} & \not 1(\bmod n) \\
e^{(i)} & \in \mathcal{O P}([\sqrt{n} / 4])(0 \leq i \leq r) \\
e^{(i)} & \neq e^{(j)}(0 \leq i<j \leq r) \\
I D^{e^{(i)} \ldots e^{(i)}} & \not \equiv I D(\bmod n)(0 \leq i<j \leq r)
\end{aligned}
$$

in the $r$-th key-updating of SKU (see Lemma 3.3.), where $n \in S \mathcal{C}_{k}, I D \in Z_{n}^{*}, S^{(r)}=$ $\left(S^{(0)}, S^{(1)}, \ldots, S^{(r)}\right)$, and $e^{(r)}=\left(e^{(0)}, e^{(1)}, \ldots, e^{(r)}\right)$. In a way similar to the above, we define $\mathcal{C}_{\mathrm{PKU}}$ to be a set of tuples $\left\langle n, I D, T^{(r)}, f^{(r)}\right\rangle$ that satisfy

$$
\begin{aligned}
I D & \equiv\left\{T^{(i)}\right\}^{(i)}(\bmod n)(0 \leq i \leq r) ; \\
I D^{2} & \neq 1(\bmod n) ; \\
f^{(i)} & \in \mathcal{O P}(\lfloor\sqrt{n} / 4 \mid)(0 \leq i \leq r) ; \\
f^{(i)} & \neq f^{(j)}(0 \leq i<j \leq r) ; \\
I D^{(j) \ldots f^{(j)}} & \neq \equiv D(\bmod n)(0 \leq i<j \leq r),
\end{aligned}
$$


in the $r$-th key-updating of PKU (see Lemma 3.4.), where $n \in S \mathcal{C}_{k}, I D \in Z_{n}^{*}, T^{(r)}=$ $\left(T^{(0)}, T^{(1)}, \ldots, T^{(r)}\right)$, and $f^{(r)}=\left(f^{(0)}, f^{(1)}, \ldots, f^{(r)}\right)$.

We use $A_{\mathrm{SKU} \rightarrow \mathrm{PKU}}$ to denote any algorithm that, on input $\left\langle n, I D, S^{(r)}, e^{(r)}\right\rangle \in \mathcal{C}_{\mathrm{SKU}}$, outputs $\left\langle n, I D, T^{(r)}, f^{(r)}\right\rangle \in \mathcal{C}_{\mathrm{PKU}}$, and $A_{\mathrm{PKU} \rightarrow \mathrm{SKU}}$ to denote any algorithm that, on input $\left\langle n, I D, T^{(r)}, f^{(r)}\right\rangle \in \mathcal{C}_{\mathrm{PKU}}$, outputs $\left\langle n, I D, S^{(r)}, e^{(r)}\right\rangle \in \mathcal{C}_{\mathrm{SKU}}$. Then we have the following theorems on deterministic polynomial time transformability between SKU and PKU.

Theorem 3.5: There exists a deterministic polynomial time algorithm $A_{\mathrm{SKU} \rightarrow \mathrm{PKU}}$.

Sketch of Proof: On input $\left\langle n, I D, S^{(r)}, e^{(r)}\right\rangle \in \mathcal{C}_{5 K U}$, the algorithm $A_{\mathrm{SKU} \rightarrow \mathrm{PKU}}$ sets $T^{(0)}:=S^{(0)}, f^{(i)}:=e^{(i)}(0 \leq i \leq r)$, and computes $T^{(i)} \equiv\left\{S^{(i)}\right\}^{(0) \ldots e^{(i-1)}},(\bmod n)$ $(1 \leq i \leq r)$. Then it outputs $\left\langle n, I D, T^{(r)}, f^{(r)}\right\rangle$, where $T^{(r)}=\left(T^{(0)}, T^{(1)}, \ldots, T^{(r)}\right), f^{(r)}=$ $\left(f^{(0)}, f^{(1)}, \ldots, f^{(r)}\right)$. It is easy to see that the algorithm $A_{\mathrm{SKU} \rightarrow \mathrm{PKU}}$ runs in deterministic polynomial time and $\left\langle n, I D, T^{(r)}, f^{(r)}\right\rangle \in \mathcal{C}_{\mathrm{PKU}}$.

Theorem 3.6: There exists a deterministic polynomial time algorithm $A_{\mathrm{PKO} \rightarrow \mathrm{SKU}}$.

Sketch of Proof: Let $n$ be an odd composite. We assume here that $x_{1}$ is the $a_{1}$-th root of $y$ modulo $n$, and $x_{2}$ is the $a_{2}$-th root of $y$ modulo $n$, where $\operatorname{gcd}\left(a_{1}, a_{2}\right)=1$ and $y \in Z_{n}^{*}$. Then we can compute $x$, the $a_{1} a_{2}$-th root of $y$ modulo $n$, by algorithm $E$ (see below.) in deterministic polynomial time without knowing prime factors of $n$. On input $n, y, x_{1}, a_{1}, x_{2}$, and $a_{2}$, the algorithm $E$ computes two integers $s$ and $t$ such that $t a_{1}+s a_{2}=1$ by Euclidean algorithm, and outputs $x \equiv x_{1}^{s} \cdot x_{2}^{t}(\bmod n)$. It is easy to see that the algorithm $E$ runs in deterministic polynomial time and $x$ is the $a_{1} a_{2}$-th root of $y$ modulo $n$. The algorithm $A_{\mathrm{PKU} \rightarrow \mathrm{SKU}}$ runs in the following way:

On input $\left\langle n, I D, T^{(r)}, f^{(r)}\right\rangle \in \mathcal{C}_{\mathrm{PKU}}$, the algorithm $A_{\mathrm{PKU} \rightarrow \mathrm{SKU}}$ sets $T^{(0)}:=S^{(0)}$, $f^{(0)}:=e^{(0)}$, and computes $x_{i}(1 \leq i \leq r)$ by running the deterministic polynomial time algorithm $E$ on input $\left\langle n, I D, S^{(i-1)}, e^{(0)} e^{(1)} \cdots e^{(i-1)}, T^{(i)}, f^{(i)}\right\rangle$. Then the algorithm $A_{\mathrm{PKU} \rightarrow \mathrm{SKU}}$ substitutes $x_{i}$ to $S^{(i)}$ and $f^{(i)}$ to $e^{(i)}(1 \leq i \leq r)$, and outputs $\left\langle n, I D, S^{(r)}, e^{(r)}\right\rangle$, where $S^{(r)}=\left(S^{(0)}, S^{(1)}, \ldots, S^{(r)}\right)$ and $e^{(r)}=\left(e^{(0)}, e^{(1)}, \ldots, e^{(r)}\right)$.

It is not difficult to see that the algorithm $A_{\mathrm{PKU} \rightarrow \mathrm{SKU}}$ runs in deterministic polynomial time and $\left\langle n, I D, S^{(r)}, e^{(r)}\right\rangle \in \mathcal{C}_{\mathrm{SKU}}$.

\section{SKU and PKU are Provably Secure}

This section shows that key-updating schemes SKU and PKU are provably secure against polynomially many times key-updating under the assumption that decrypting RSA is hard 
for $n \in \mathcal{S C}_{k}$, i.e., even if any polynomially many entities conspire, they can not find a secret-key of any other entity in polynomially many times key-updating.

To show this, we provide several lemmas in the following:

Lemma 4.1: Let $n \in \mathcal{S C}_{k}$ and let $U(|n|)$ be any fixed polynomial in $|n|$. Let $r$ be any positive integer not greater than $U(|n|)$ and let $e^{(i)}<\lfloor\sqrt{n} / 4\rfloor(0 \leq i \leq r-1)$ be distinct $r$ odd primes. Then the probability $P$ that for any $d<\lfloor\sqrt{n} / 4\rfloor, d \in \mathcal{O P}(\lfloor\sqrt{n} / 4\rfloor)$ and $d \neq e^{(i)}(0 \leq i \leq r-1)$ is greater than $C /|n|$ for some $C>0$ and sufficiently large $n$.

Proof: Let $\pi(x)$ denote the number of primes not greater than $x(x \geq 2)$. From prime number theorem [HW], it follows that

$$
\pi(x)>C_{0} \frac{x}{\log _{2} x},
$$

for some constant $C_{0}$. Then the probability $P$ is

$$
\begin{aligned}
P & >\frac{C_{0} \frac{\lfloor\sqrt{n} / 4\rfloor-1}{\log _{2}(\lfloor\sqrt{n} / 4\rfloor-1)}-(r+1)}{\lfloor\sqrt{n} / 4\rfloor-1}>\frac{C_{1}}{\log _{2}\lfloor\sqrt{n} / 4\rfloor}-\frac{r+1}{\lfloor\sqrt{n} / 4\rfloor-1} \\
> & \frac{C_{1}}{\log _{2}\lfloor\sqrt{n} / 4\rfloor}-\frac{2 U(|n|)}{\lfloor\sqrt{n} / 4\rfloor-1}>\frac{C_{2}}{\log _{2}\lfloor\sqrt{n} / 4\rfloor} \\
> & \frac{C_{3}}{\log _{2}(\sqrt{n} / 4)}>\frac{C_{4}}{\left\lfloor\log _{2} n\right\rfloor+1},
\end{aligned}
$$

thus $P>C /|n|$ for some constant $C$ and sufficiently large $n$.

Lemma 4.2: Let $n \in S \mathcal{C}_{k}$ and let $U(|n|)$ be any fixed polynomial in $|n|$. Let $r$ be any positive integer not greater than $U(|n|)$ and let $e^{(r-1)}=\left(e^{(0)}, e^{(1)}, \ldots, e^{(r-1)}\right)$, where $e^{(i)}<$ $\lfloor\sqrt{n} / 4\rfloor(0 \leq i \leq r-1)$ are distinct $r$ odd primes. Define $\hat{e}^{(r)}=\left(\hat{e}^{(0)}, \hat{e}^{(1)}, \ldots, \hat{e}^{(r)}\right)$ to be $\hat{e}^{(r)}=\left(e^{(0)}, e^{(1)}, \ldots, e^{(r-1)}, d\right)$ for any $d \in \mathcal{O P}(\lfloor\sqrt{n} / 4\rfloor)$ such that $d \neq e^{(i)}(0 \leq i \leq r-1)$. Then for any $g \in Z_{n}^{*}$ such that $g^{2} \not \equiv 1(\bmod n), g^{\dot{(i)}^{(i)} \dot{e}^{(i)}} \neq g(\bmod n)(0 \leq i<j \leq r)$ iff $g^{e^{(t)} \cdots e^{(i)}} \not \equiv g(\bmod n)(0 \leq s<t \leq r-1)$ and $\left\{\prod_{l=i}^{r-1} e^{(\ell)}\right\} \cdot d \neq 1(\bmod L)(0 \leq i \leq r-1)$, where $L$ is the order of $g$ modulo $n$.

Proof: Let $L$ denote the order of $g$ modulo $n$. Then it is clear that

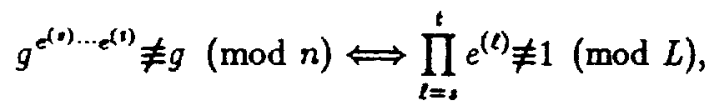

for all $s, t(0 \leq s<t \leq r-1)$, and

$$
g^{\dot{\varepsilon}^{(i)} \ldots \dot{e}^{(j)}} \not \equiv g(\bmod n) \Longleftrightarrow \prod_{\ell=i}^{j} \hat{e}^{(l)} \not 1(\bmod L),
$$


for all $i, j(0 \leq i<j \leq r)$. Thus it suffices to show that

$$
\begin{aligned}
\prod_{\ell=i}^{j} \hat{e}^{(\ell)} \neq 1 & (\bmod L)(0 \leq i<j \leq r) \\
\Longleftrightarrow & \prod_{\ell=s}^{t} e^{(\ell)} \neq 1(\bmod L)(0 \leq s<t \leq r-1) \\
& \bigwedge\left\{\prod_{\ell=i}^{r-1} e^{(\ell)}\right\} \cdot d \not \neq 1(\bmod L)(0 \leq i \leq r-1) .
\end{aligned}
$$

When $j<r, \prod_{\ell=i}^{j} \hat{e}^{(\ell)} \not \equiv 1(\bmod L)(0 \leq i<j \leq r-1)$ iff $\prod_{\ell=s}^{t} e^{(\ell)} \not \equiv 1(\bmod L)(0 \leq s<$ $t \leq r-1)$, and when $j=r, \prod_{\ell=i}^{j} \hat{e}^{(\ell)} \not \equiv 1(\bmod L)(0 \leq i \leq r-1)$ iff $\left\{\prod_{\ell=i}^{r-1} e^{(\ell)}\right\} \cdot d \not \equiv 1$ $(\bmod L)(0 \leq i \leq r-1)$. Thus it is immediate to see that $g^{\hat{e}^{(i)} \ldots e^{(j)}} \neq \equiv(\bmod n)(0 \leq$ $i<j \leq r)$ iff $g^{e^{(t) \ldots} e^{(t)}} \not \equiv g(\bmod n)(0 \leq s<t \leq r-1)$ and $\left\{\prod_{l=i}^{r-1} e^{(\ell)}\right\} \cdot d \neq 1(\bmod L)$ $(0 \leq i \leq r-1)$.

For $n \in \mathcal{S C}_{k}$ and $e^{(i)} \in \mathcal{O P}(\lfloor\sqrt{n} / 4\rfloor)(0 \leq i \leq r-1)$ such that $e^{(i)} \neq e^{(j)}(0 \leq i<j \leq$ $r-1)$, we define $\mathcal{D}_{r+1}$ to be a set of $(r+1)$ distinct $d_{j} \in \mathcal{O P}(\lfloor\sqrt{n} / 4\rfloor)(1 \leq j \leq r+1)$ such that $d_{j} \neq e^{(i)}(1 \leq j \leq r+1,0 \leq i \leq r-1)$.

Lemma 4.3: Let $n \in \mathcal{S C}_{k}$ and let $U(|n|)$ be any fixed polynomial in $|n|$. Let $r$ be any positive integer not greater than $U(|n|)$ and let $e^{(r-1)}=\left(e^{(0)}, e^{(1)}, \ldots, e^{(r-1)}\right)$, where $e^{(i)}<\lfloor\sqrt{n} / 4\rfloor(0 \leq i \leq r-1)$ are distinct $r$ odd primes. Then for any $g \in Z_{n}^{*}$ such that $g^{2} \not \equiv 1(\bmod n)$, there exists at least one $d \in \mathcal{D}_{r+1}$ such that $\left\{\prod_{\ell=i}^{r-1} e^{(\ell)}\right\} \cdot d \not \equiv 1(\bmod L)$ for any $i(0 \leq i \leq r-1)$, where $L$ is the order of $g$ modulo $n$.

Proof: Let $L$ denote the order of $g$ modulo $n$. Since $n \in S \mathcal{C}_{k}$ and $g\left(\in Z_{n}^{*}\right)$ satisfies $g^{2} \not \equiv 1$ $(\bmod n), L \geq \min \left\{p^{\prime}, q^{\prime}\right\}$. From Lemma 3.1 , it follows that $\lfloor\sqrt{n} / 4\rfloor \leq \min \left\{p^{\prime}, q^{\prime}\right\}$, and thus for any $d<\lfloor\sqrt{n} / 4\rfloor, d<L$. For some $i(0 \leq i \leq r-1)$, there exist at most $r$ distinct $d_{j}<\lfloor\sqrt{n} / 4\rfloor$ that satisfy $\left\{\prod_{\ell=i}^{r-1} e^{(\ell)}\right\} \cdot d_{j} \equiv 1(\bmod L)$, hence at least one $d \in \mathcal{D}_{r+1}$ must satisfy $\left\{\prod_{\ell=i}^{r-1} e^{(\ell)}\right\} \cdot d \not \equiv 1(\bmod L)$ for any $i(0 \leq i \leq r-1)$.

For simplicity, we assume that every entity $i$ is numbered as $1,2, \ldots$ Let $E(|n|)$ and $U(|n|)$ be any fixed polynomials in $|n|$. When $m(<E(|n|))$ entities, each of which is in the $r_{i}$-th $\left(1 \leq r_{i} \leq U(|n|)\right)$ key-updating, conspire to find a secret-key of any other entity $u(>m)$, they can use the following information in SKU.

$$
\begin{aligned}
& n \in \mathcal{S} \mathcal{C}_{k} ; e_{u}^{\left(r_{u}\right)}=e_{0}^{\prime} e_{1}^{\prime} \cdots e_{r_{u}}^{\prime}, e_{i}^{\prime} \in \mathcal{O P}(\lfloor\sqrt{n} / 4\rfloor)\left(0 \leq i \leq r_{u}\right) ; \\
& I D_{u} \in \mathcal{Z}_{n}^{*}\left(I D_{u}^{2} \not \equiv 1(\bmod n)\right) ;\left(n, I D_{i}, S_{i}^{\left(r_{i}\right)}, e_{i}^{\left(r_{i}\right)}\right) \in \mathcal{C}_{\text {SKU }}(1 \leq i \leq m) \text {, }
\end{aligned}
$$


where $\boldsymbol{S}_{i}^{\left(r_{i}\right)}=\left(S_{i}^{(0)}, S_{i}^{(1)}, \ldots, S_{i}^{\left(r_{i}\right)}\right), \boldsymbol{e}_{i}^{\left(r_{i}\right)}=\left(e_{i}^{(0)}, e_{i}^{(1)}, \ldots, e_{i}^{\left(r_{i}\right)}\right)(1 \leq i \leq m)$. Let $R$ be $m$ tuple of integers, $R=\left(r_{1}, r_{2}, \ldots, r_{m}\right)$, and each $r_{i}(1 \leq i \leq m)$ is not greater than $U(|n|)$. Then we use $I N V_{\mathrm{SKU}}^{(m, R)}$ to denote any algorithm that, on input

$$
\begin{gathered}
n \in \mathcal{S C}_{k} ; e_{u}^{\left(r_{*}\right)}=e_{0}^{\prime} e_{1}^{f} \cdots e_{\tau_{v}}^{\prime}, e_{i}^{\prime} \in \mathcal{O P}(\lfloor\sqrt{n} / 4\rfloor)\left(0 \leq i \leq r_{u}\right) \\
x \in \mathcal{Z}_{n}^{*}\left(x^{2} \not \equiv 1(\bmod n)\right) ;\left\langle n, I D_{i}, S_{i}^{\left(r_{i}\right)}, e_{i}^{\left(r_{i}\right)}\right\rangle \in \mathcal{C}_{\mathrm{SKU}}(1 \leq i \leq m),
\end{gathered}
$$

outputs $y \in \mathcal{Z}_{n}^{*}$ such that $x \equiv y^{e}(\bmod n)$ for a non-negligible fraction of $x \in \mathcal{Z}_{n}^{*}$, and we use $I N V_{\mathrm{PKU}}^{(m, R)}$ to denote any algorithm that, on input

$$
\begin{gathered}
n \in \mathcal{S C}_{k} ; f \in \mathcal{O P}(\lfloor\sqrt{n} / 4\rfloor) ; x \in \mathcal{Z}_{n}^{*}\left(x^{2} \not \equiv 1(\bmod n)\right) \\
\left\langle n, I D_{i}, T_{i}^{\left(r_{i}\right)}, f_{i}^{\left(r_{i}\right)}\right\rangle \in \mathcal{C}_{\mathrm{PKU}}(1 \leq i \leq m),
\end{gathered}
$$

outputs $y \in \mathcal{Z}_{n}^{*}$ such that $x \equiv y^{f}(\bmod n)$ for a non-negligible fraction of $x \in \mathcal{Z}_{n}^{*}$, where $\boldsymbol{T}=\left(T_{i}^{(0)}, T_{i}^{(1)}, \ldots, T_{i}^{\left(r_{i}\right)}\right), f_{i}^{\left(r_{i}\right)}=\left(f_{i}^{(0)}, f_{i}^{(1)}, \ldots, f_{i}^{\left(r_{i}\right)}\right)(1 \leq i \leq m)$. In addition, we use $I N V$ to denote any algorithm that, on input $n \in \mathcal{S C}_{k}, e \in \mathcal{O P}(\lfloor\sqrt{n} / 4\rfloor)$, and $x \in \mathcal{Z}_{n}^{*}$, outputs $y \in \mathcal{Z}_{n}^{*}$ such that $x \equiv y^{*}(\bmod n)$ for a non-negligible fraction of $x \in \mathcal{Z}_{n}^{*}$.

From technical reasons, we assume, throughout the rest of this paper, that each $I D_{i}$ such that $I D_{i}^{2} \not \equiv 1(\bmod n)$ is randomly chosen (by the trusted center) with uniform probability over $\mathcal{Z}_{n}^{*}$, but once assigned it is unchanged forever.

Theorem 4.4: Given an expected polynomial time algorithm $I N V_{\mathrm{SKU}}^{(m, R)}$, there exists an expected polynomial time algorithm $I N V$ using $I N V_{\mathrm{SKU}}^{(m, R)}$ as an oracle.

Proof: It suffices to show that, given $n \in \mathcal{S C}_{k}$, $e=e_{0}^{\prime} e_{1}^{\prime} \cdots e_{r_{8}}^{\prime}$, and $x \in \mathcal{Z}_{n}^{*}$ such that $x^{2} \not \equiv 1(\bmod n)$, there exists an expected polynomial time $I N V\left(I N V_{\mathrm{SKU}}^{(m, R)}\right)$ using an expected polynomial time algorithm $I N V_{\mathrm{SKU}}^{(m, R)}$ as an oracle.

Let $E(|n|)$ and $U(|n|)$ be any fixed polynomials in $|n|$. Let $m<E(|n|)$ and let $R=\left(r_{1}, r_{2}, \ldots, r_{m}\right)$, where $r_{i} \leq U(|n|)(1 \leq i \leq m)$. Note that for $\left\langle n, I D_{i}, S_{i}^{\left(r_{i}\right)}, \boldsymbol{e}_{i}^{\left(r_{i}\right)}\right\rangle$ $(1 \leq i \leq m)$, the oracle $I N V_{\mathrm{SKU}}^{(m, R)}$ returns a correct answer if $\left\langle n, I D_{i}, \boldsymbol{S}_{i}^{\left(\boldsymbol{r}_{i}\right)}, \boldsymbol{e}_{i}^{\left(r_{i}\right)}\right\rangle \in \mathcal{C}_{\mathrm{SKU}}$ for all $i(1 \leq i \leq m)$; it might return garbage or something otherwise.

Algorithm $I N V\left(I N V_{\mathrm{SKU}}^{(m, R)}\right)$ :

Input. $\quad n \in \mathcal{S C}_{k} ; e=e_{0}^{\prime} e_{1}^{\prime} \cdots e_{r_{u}}^{\prime}$, where $e_{i}^{\prime} \in \mathcal{O P}(\lfloor\sqrt{n} / 4\rfloor)\left(0 \leq i \leq r_{u}\right)$; $x \in \mathcal{Z}_{n}^{*}$ such that $x^{2} \not \equiv 1(\bmod n)$.

Step 1. Set $\mathcal{R}:=\phi$ and $i:=1$.

Step 2. Set $S_{i}:=\phi$ and $\ell_{i}=r_{i}$ and choose $S_{i}^{\left(r_{i}\right)} \in \mathcal{Z}_{n}^{*}$ such that $\left\{S_{i}^{\left(r_{i}\right)}\right\}^{2} \not \equiv 1(\bmod n)$.

Step 3. Choose $\left(r_{i}-\ell_{i}+1\right)$ distinct $d, \in \mathcal{O} \mathcal{P}(\lfloor\sqrt{n} / 4\rfloor)$ such that $d_{j} \notin \mathcal{S}_{i}$, using primality testing. (see, e.g., [AH], [Ra], [SS].) 
Step 4. For each $d, \notin \mathcal{S}_{i}\left(1 \leq j \leq r_{i}-\ell_{i}+1\right)$, compute $S_{i}^{\left(\ell_{i}-1\right)} \equiv\left\{S_{i}^{\left(\ell_{i}\right)}\right\}^{d_{j}}(\bmod n)$ until $S_{i}^{\left(\ell_{i}-1\right)} \not S_{i}^{(\ell)}(\bmod n)$ for all $\ell\left(\ell_{i} \leq \ell \leq r_{i}\right)$.

Step 5. Set $e_{i}^{\left(\ell_{i}\right)}:=d_{j}$ and $\mathcal{S}_{i}:=S_{i} \cup e_{i}^{\left(\ell_{i}\right)}$.

Step 6. If $\ell_{i}>0$, then $\ell_{i}:=\ell_{i}-1$ and go to Step 3 .

Step 7. Set $I D_{i}:=S_{i}^{(-1)}$.

Step 7-1. If $I D_{i} \in \mathcal{R}$, then go to Step 2; otherwise set $S_{i}^{\left(r_{i}\right)}=\left(S_{i}^{(0)}, S_{i}^{(1)}, \ldots, S_{i}^{\left(r_{i}\right)}\right)$ and $\boldsymbol{e}_{i}^{\left(r_{i}\right)}=\left(e_{i}^{(0)}, e_{i}^{(1)}, \ldots, e_{i}^{\left(r_{i}\right)}\right)$.

Step 7-2. If $i<m$, then $\mathcal{R}:=\mathcal{R} \cup I D_{i}, i:=i+1$ and go to Step 2.

Step 8. Run the algorithm (or oracle) $I N V_{\mathrm{SKU}}^{(m, R)}$ on input $n \in S \mathcal{C}_{k}, e=e_{0}^{\prime} e_{1}^{\prime} \cdots e_{\tau_{*}}^{\prime}$, $x \in \mathcal{Z}_{n}^{*}$ such that $x^{2} \not \equiv 1(\bmod n)$, and $\left\langle n, I D_{i}, S_{i}^{\left(r_{i}\right)}, e_{i}^{\left(r_{i}\right)}\right\rangle(1 \leq i \leq m)$.

Output. $y \in \mathcal{Z}_{n}^{*}$ such that $x \equiv y^{e}(\bmod n)$.

Trivially, Step 1 (resp. Step 2) runs in deterministic (resp. expected) polynomial time. From Lemma 4.1 and the facts that deciding primality is in $\mathcal{Z P P}$ (see [AH], [Ra], [SS].) and $\left(r_{i}-\ell_{i}+1\right) \leq U(|n|)+1$, it follows that Step 3 runs in expected polynomial time. From Lemmas 4.2 and 4.3 , there must exist at least one $d_{j} \notin \mathcal{S}_{i}$ such that for all $\ell$ $\left(\ell_{i} \leq \ell \leq r_{i}\right), S_{i}^{\left(\ell_{i}-1\right)} \not \equiv S_{i}^{(\ell)}(\bmod n)$, thus Step 4 runs in deterministic polynomial time. Since Step 5 runs in deterministic polynomial time and the iteration times of a loop from Step 3 to 6 is $r_{i}+1 \leq U(|n|)+1$, then the total running cost of the loop from Step 3 to 6 is expected polynomial time.

In Step 7-1, the probability that $I D_{i} \in \mathcal{R}$ is negligibly small, because possible candidates of $I D_{i}$ is exponentially many, while $\|\mathcal{R}\|$ is polynomially bounded, i.e., $\|\mathcal{R}\| \leq m<$ $E(|n|)$, and then the expected iteration times from Step 2 to 7-1 or 7-2 is $O(E(|n|))$. Hence the algorithm $I N V\left(I N V_{\mathrm{SKU}}^{(m, R)}\right)$ runs in expected polynomial time and outputs $y \in \mathcal{Z}_{n}^{*}$ such that $y \equiv x^{e}(\bmod n)$ for a non-negligible fraction of $x \in \mathcal{Z}_{n}^{*}$.

Informally, Theorem 4.4 shows that when any polynomially many entities conspire in SKU even in polynomially many times key-updating, they can not invert $x \in \mathcal{Z}_{n}^{*}$ for a non-negligible fraction of $x \in \mathcal{Z}_{n}^{*}$. From the definition of soundness [FFS], [TW], this implies that in SKU any polynomially many conspiring entities can not misrepresent themselves for a non-negligible fraction of (possible) other entities, even in polynomially many times key-updating. A result similar to this holds for PKU.

Theorem 4.5: Given an expected polynomial time algorithm $I N V_{\mathrm{PKU}}^{(m, R)}$, there exists an expected polynomial time algorithm $I N V$ using $I N V_{\mathrm{PKU}}^{(m, R)}$ as an oracle.

Proof: It suffices to show that, given $n \in \mathcal{S C} \mathcal{C}_{k}, f \in \mathcal{O P}(\lfloor\sqrt{n} / 4\rfloor)$, and $x \in \mathcal{Z}_{n}^{*}$ such 
that $x^{2} \not \equiv 1(\bmod n)$, there exists an expected polynomial time $I N V\left(I N V_{\mathrm{PKU}}^{(m, R)}\right)$ using an expected polynomial time algorithm $I N V_{\mathrm{PKU}}^{(m, R)}$ as an oracle.

Let $E(|n|), U(|n|), m$, and $R=\left(r_{1}, r_{2} \ldots, r_{m}\right)$ be defined in the same way as the proof of Theorem 4.4. It should be noted that for $\left\langle n, I D_{i}, \boldsymbol{T}_{i}^{\left(r_{i}\right)}, f_{i}^{\left(r_{i}\right)}\right\rangle(1 \leq i \leq m)$, the oracle $I N V_{\mathrm{PKU}}^{(m, R)}$ returns a correct answer if $\left\langle n, I D_{i}, T_{i}^{\left(r_{i}\right)}, f_{i}^{\left(r_{i}\right)}\right\rangle \in \mathcal{C}_{\mathrm{PKU}}$ for all $i(1 \leq i \leq m)$; it might return garbage or something otherwise.

Algorithm $I N V\left(I N V_{\mathrm{PKU}}^{\left(m_{1} R\right)}\right)$ :

Input. $\quad n \in \mathcal{S C}_{k} ; f \in \mathcal{O P}(\lfloor\sqrt{n} / 4\rfloor) ; x \in \mathcal{Z}_{n}^{*}$ such that $x^{2} \not \equiv 1(\bmod n)$.

Step 1. Run each Step from 1 to 7 in the algorithm $I N V\left(I N V_{\mathrm{SKU}}^{(m, R)}\right)$.

Step 2. Run the algorithm $A_{\mathrm{SKU} \rightarrow \mathrm{PKU}}$ on input $\left\langle n_{,} I D_{i}, \boldsymbol{S}_{i}^{\left(r_{i}\right)}, \boldsymbol{e}_{i}^{\left(r_{i}\right)}\right\rangle \in \mathcal{C}_{\mathrm{SKU}}$ for each $i$ $(1 \leq i \leq m)$, and output $\left\langle n, I D_{i}, T_{i}^{\left(r_{i}\right)}, f_{i}^{\left(r_{i}\right)}\right\rangle \in \mathcal{C}_{\mathrm{PKU}}$.

Step 3. Run the algorithm (oracle) $I N V_{\mathrm{PKU}}^{(m, R)}$ on input $n \in \mathcal{S C}_{k}, f \in \mathcal{O P}(\lfloor\sqrt{n} / 4\rfloor)$, $x \in \mathcal{Z}_{n}^{*}$ such that $x^{2} \not \equiv 1(\bmod n)$, and $\left\langle n, I D_{i}, T_{i}^{\left(r_{i}\right)}, f_{i}^{\left(r_{i}\right)}\right\rangle(1 \leq i \leq m)$.

Output. $y \in \mathcal{Z}_{n}^{*}$ such that $x \equiv y^{f}(\bmod n)$.

From the proof of Theorem 4.4, it follows that Step 1 runs in expected polynomial time. Since $m<E(|n|)$ and Theorem 3.5 guarantees that the algorithm $A_{\mathrm{SKU} \rightarrow \mathrm{PKU}}$ runs in deterministic polynomial time, Step 2 runs in deterministic polynomial time.

Hence the algorithm $I N V\left(I N V_{S K U}^{(m, R)}\right)$ runs in expected polynomial time and outputs $y \in \mathcal{Z}_{n}^{*}$ such that $y \equiv x^{f}(\bmod n)$ for a non-negligible fraction of $x \in \mathcal{Z}_{n}^{*}$.

\section{Conclusion and Remarks}

In this paper, we showed two kinds of secure key-updating schemes SKU and PKU in the extended Fiat-Shamir scheme. Here we define more general schemes SKU' and PKU':

Let $n \in \mathcal{S C}_{k}$ and let $E(|n|)$ and $U(|n|)$ be any fixed polynomial in $|n|$. Then the key-updating scheme SKU' is completely the same as SKU except that for each entity $i, e_{i}^{(j)}<\lfloor\sqrt{n} / 4\rfloor\left(0 \leq j \leq r_{i} \leq U(|n|)\right)$ is an odd number and is not necessarily distinct from each other, and the key-updating scheme PKU' is also completely the same as PKU except that for each entity $i, f_{i}^{(j)} \not f_{i}^{(k)}\left(0 \leq k<j \leq r_{i}\right)$. Using a technique similar to the proofs of Theorems 4.4 and 4.5, we can show that both SKU' and PKU' are provably secure if decrypting RSA is hard for $n \in \mathcal{S C}_{k}$.

Observing the results in this paper, we can say that SKU and PKU have the same security with each other in a polynomial time sense, and seemingly so do SKU' and PKU'. The scheme PKU, however, seems to be better one than SKU in the light of 
efficiency, because in the $r_{i}$-th $\left(1 \leq r_{i} \leq U(|n|)\right)$ key-updating of PKU, a public-key of each entity $i$ is only a prime $f_{i}^{\left(r_{i}\right)}$, while in the $r_{i}$-th $\left(1 \leq r_{i} \leq U(|n|)\right)$ key-updating of SKU, a public-key of each entity $i$ is $\prod_{j=0}^{r_{i}} e_{i}^{(j)}$. This is also the case for PKU' and SKU'.

Our results can be generalized to more theoretical form - For any transitive trapdoor random self-reducible uniform relation (see [IST].), there exists a perfect zero-knowledge (identity-based) identification system with provably secure key-updating schemes, i.e., if any polynomially many entities conspire in polynomially many times key-updating, they can not find a secret-key of a non-negligible fraction of (possible) other entities, or they can not misrepresent themselves for a non-negligible fraction of (possible) other entities.

\section{Acknowledgments}

The authors wish to thank Mitsunori Ogiwara, Yuliang Zheng, Kouichi Sakurai, Kenji Koyama, Tsutomu Matsumoto, and Hiroki Shizuya for their valuable comments and suggestions. The authors also would like to thank Osamu Watanabe for his cooperative and suggestive discussion on the early version of this work.

\section{References}

[AH] Adleman, L.M. and Huang, M.D.A., "Recognizing Primes in Random Polynomial Time," Proc. of 19 th Annual ACM Symposium on Theory of Computing, pp.462469 (May, 1987).

[AL] Angluin, D. and Lichtenstein, D., "Provable Security of Cryptosystems: a Survey," Technical Report TR-288, Yale University (October, 1983).

[FFS] Feige, U., Fiat, A., and Shamir, A., "Zero Knowledge Proofs of Identity," Journal of Cryptology, Vol.1, No.1, pp.77-94 (1988).

[FS] Fiat, A. and Shamir, A., "How to Prove Yourself: Practical Solutions to Identification and Signature Problems," in Advances in Cryptology - Crypto'86, Lecture Notes in Computer Science 263, Springer-Verlag, Berlin, pp.186-194 (1987).

[FT] Fujioka, A. and Tsujii, S., "An ID-Based Identification System with Simple KeyUpdating," Technical Report of IEICE, ISEC89-25 (November, 1989).

[GQ] Guillou, L.C. and Quisquater, J.J., "A Practical Zero-Knowledge Protocol Fitted to Security Microprocessors Minimizing both Transmission and Memory," in Advances 
[GQ] Guillou, L.C. and Quisquater, J.J., "A Practical Zero-Knowledge Protocol Fitted to Security Microprocessors Minimizing both Transmission and Memory," in Advances in Cryptology - Eurocrypt'88, Lecture Notes in Computer Science 330, Springer-Verlag, Berlin, pp.123-128 (1988).

[HW] Hardy, G.H. and Wright, E.M., An Introduction to the Theory of Numbers, Oxford University Press, 5th Edition (1979).

[IST] Itoh, T., Shinozaki, S., and Tsujii, S., "Secure Key-Updating Schemes in Identifcation Protocols," manuscript (March, 1990).

[Kr] Kranakis, E., Primality and Cryptography, Wiley-Teubner Series in Computer Science, John Wiley \& Sons, Chishester (1986).

[OO] Ohta, K. and Okamoto, T., "A Modification of the Fiat-Shamir Scheme," in Advances in Cryptology - Crypto'88, Lecture Notes in Computer Science 403, Springer-Verlag, Berlin, pp.232-243 (1989).

[Ra] Rabin, M.O., "Probabilistic Algorithm for Primality Testing," Journal of Number Theory, Vol.12, pp.128-138 (1980).

[RSA] Rivest, R.L., Shamir, A., and Adleman, L.M., "A Method for Obtaining Digital Signatures and Public-Key Cryptosystems," Communication of the ACM, Vol.21, No.2, pp.120-126 (February, 1978).

[SI] Shizuya, H. and Itoh, T., "A Group-Theoretic Interface to Random Self-Reducibility," to appear in The Transactions of the IEICE, Vol.E 73, No.7 (July, 1990).

[SS] Solovay, R. and Strassen, V., "A Fast Monte Calro Test for Primality," SIAM Journal on Computing, Vol.6, No.1, pp.84-85 (March, 1977).

[TW] Tompa, M. and Woll, H., "Random Self-Reducibility and Zero Knowledge Interactive Proofs of Possession of Information," Proc. of 28 th Annual IEEE Symposium on Foundations of Computer Science, pp.472-482 (October, 1987). 